\title{
High-resolution Laue-type DuMond curved crystal spectrometer
}

\author{
M. Szlachetko, ${ }^{1, a)}$ M. Berset, ${ }^{1}$ J.-Cl. Dousse, ${ }^{1}$ J. Hoszowska, ${ }^{1}$ and J. Szlachetko ${ }^{2,3, b)}$ \\ ${ }^{1}$ Department of Physics, University of Fribourg, CH-1700 Fribourg, Switzerland \\ ${ }^{2}$ Paul Scherrer Institute, $\mathrm{CH}-5232$ Villigen PSI, Switzerland \\ ${ }^{3}$ Institute of Physics, Jan Kochanowski University, 25-406 Kielce, Poland
}

\begin{abstract}
We report on a high-resolution transmission-type curved crystal spectrometer based on the modified DuMond slit geometry. The spectrometer was developed at the University of Fribourg for the study of photoinduced X-ray spectra. $K$ and $L$ X-ray transitions with energies above about $10 \mathrm{keV}$ can be measured with an instrumental resolution comparable to their natural linewidths. Construction details and operational characteristics of the spectrometer are presented. The variation of the energy resolution as a function of the focal distance and diffraction order is discussed. The high sensitivity of the spectrometer is demonstrated via the $2 s-1 s$ dipole-forbidden X-ray transition of Gd which could be observed despite its extremely low intensity. The precision of the instrument is illustrated by comparing the sum of the energies of the Au $K-L_{2}$ and $L_{2}-M_{3}$ cascading transitions with the energy of the crossover $K-M_{3}$ transition as well as by considering the energy differences of the $\mathrm{Gd}$ $K \alpha_{1} \mathrm{X}$-ray line measured at five different diffraction orders. Finally, to demonstrate the versatility of the spectrometer, it is shown that the latter can also be used for in-house extended X-ray absorption fine structure measurements.
\end{abstract}

\section{INTRODUCTION}

For the detection of X-rays or $\gamma$-rays energy dispersive or wavelength dispersive instruments can be used. The wavelength dispersive instruments (or crystal spectrometers) are characterized by a high resolving power of the order of $10^{-4}$ which is much better than the one of energy-dispersive instruments $\left(\sim 10^{-1}-10^{-2}\right)$, a high precision and a good sensitivity. Unfortunately crystal spectrometers are also characterized by a rather poor efficiency which is due mainly to the limited solid angle under which the crystal is seen by the source of radiation.

Many different types of crystal spectrometers have been developed so far. Flat crystal spectrometers provide the highest resolution and precision but their luminosity is extremely weak. To increase the solid angle, the crystal can be bent, cylindrically or spherically. Bending the crystal results in luminosity enhancements of the order of $10^{2}-10^{3}$ with, however, some losses in the resolution, due to the geometrical aberrations related to imperfections in the crystal curvature and the quasi-mosaicity induced in the crystal lamina by the bending torque. For both flat and curved crystal spectrometers, Laue type (transmission type) and Bragg type (reflection type) instruments do exist. Most reflection type curved crystal spectrometers are operated in the Johann, ${ }^{1}$ Johansson, ${ }^{2}$ or von Hamos ${ }^{3-5}$ geometry. For transmission type curved crystal spectrometers the DuMond ${ }^{6}$ and Cauchois ${ }^{7}$ geometries are the most commonly employed.

In Bragg type spectrometers the diffraction planes are parallel to the surface of the crystal exposed to the incoming radiation, whereas for Laue type spectrometers the diffraction planes are normal to the exposed surface. In the Laue case the

\footnotetext{
a)Present address: Paul Scherrer Institute, CH-5232 Villigen PSI, Switzerland.

b) Electronic mail: jakub.szlachetko@psi.ch
}

photons have thus to travel through the crystal thickness to reach the detector. As a result, the intensity of the diffracted radiation is attenuated, being partly absorbed by the crystal. This absorption grows drastically when the photon energy decreases. For instance, for a $1 \mathrm{~mm}$ thick quartz plate, the absorption amounts to about $99 \%$ at $10 \mathrm{keV}$. For this reason, transmission type crystal spectrometers are usually employed for hard X-rays and $\gamma$-rays above about $20 \mathrm{keV}$. On the other hand, due to geometrical constraints related to the spectrometer design, the minimum Bragg angles sustainable by reflection type crystal spectrometers cannot be below about $20^{\circ}$. For standard crystals such as quartz, silicon, and germanium with typical spacing constants $2 d \approx 3-5 \AA$, Bragg angles $\theta \geq 20^{\circ}$ correspond to photon energies $\mathrm{E} \leq 7-12 \mathrm{keV}$. As in addition, the large penetration depth of high energy photons leads in the Bragg case to a broadening of the measured $\mathrm{X}$-ray lines, reflection type crystal spectrometers are generally used for measurements of soft and tender X-rays up to about $10 \mathrm{keV}$.

Curved crystal spectrometers of transmission type have proven to be an important tool in X-ray spectroscopy of transitions to the $K$-shell and $L$-subshells of heavy elements. For the metrology of X-ray transitions, for which the natural linewidths, energies, and relative intensities of the transitions are of interest, an instrumental broadening comparable to the widths of the atomic levels involved in the transitions and a relative precision of $\sim 10^{-5}$ are indeed needed. Furthermore, in the $K$ and $L \mathrm{X}$-ray spectra of multiply ionized heavy atoms, the radiative decay of the multiple inner-shell vacancy states leads to satellite X-ray lines which can be only resolved from their parent diagram lines by means of high-resolution instruments such as Laue type crystal spectrometers.

A major progress in determining the energy and intensity of complex X-ray and $\gamma$-ray spectra was made by DuMond ${ }^{6}$ in 1947 with the development of a line source curved crystal 
transmission spectrometer. The DuMond geometry, in which the crystal is bent cylindrically, requires that the radius of curvature of the crystal is equal to the diameter of the so-called Rowland (or focal) circle and that the center of the radiation source and the center of the crystal are located on opposite sides of this circle. If these geometrical conditions are fulfilled, then for a particular photon energy the Bragg law is nearly satisfied over the whole crystal area exposed to the incoming radiation.

Since this pioneering work many spectrometers based on the DuMond geometry have been constructed (see, e.g., Refs. 8-16). In most of them the radiation source was no longer a $\gamma$-ray radioactive source as used by DuMond but a solid, liquid, or gaseous sample placed in the beam line of a nuclear reactor or particle accelerator. Laue-type crystal spectrometers have been installed at nuclear reactors for, e.g., high-resolution $n-\gamma$ spectroscopy experiments ${ }^{17}$ and at particle accelerators for the study of, e.g., $(\alpha, \mathrm{xn} \gamma)$ nuclear reactions, ${ }^{18}$ heavy-ion induced multiple atomic innershell ionization, ${ }^{19-21}$ or X-rays from muonic ${ }^{22}$ and pionic atoms. ${ }^{23}$ More recently, the advent of third generation synchrotron radiation (SR) facilities and X-ray free electron laser (XFEL) sources have given a new boost to the domain and many XES (X-ray emission spectroscopy) and RIXS (resonant inelastic X-ray scattering) experiments have been carried out at such facilities using crystal spectrometers. However, due the limited space available at SR and XFEL beamlines most measurements were performed with Bragg-type crystal spectrometers, ${ }^{24-32}$ only a few of them being carried out with Laue-type analyzers equipped with short focal distance bent crystals. $^{33,34}$

Thanks to the considerable developments made during the last decades in X-ray optics, electronics and informatics modern DuMond crystal spectrometers are now equipped with precise crystal bending devices, sophisticated detector systems, highly accurate laser interferometers to determine the Bragg angles, and most instruments are automatized and operated via dedicated personal computers.

In this paper we present the high-resolution DuMond type curved crystal spectrometer installed at the University of Fribourg, Switzerland. The spectrometer which was constructed for high-resolution studies of photoinduced X-ray spectra can be used for photon energies above about $10 \mathrm{keV}$. The spectrometer design and construction details including the target chamber, the crystal bending device and rotation mechanism, optical laser interferometer, collimator, detector, and driving and acquisition electronics are presented. The operational characteristics of the spectrometer and the potential of the instrument in different areas of X-ray research are illustrated by several examples of typical applications.

\section{SPECTROMETER DESIGN}

\section{A. Overview of the instrument}

In the standard DuMond geometry the source of radiation has the shape of a vertical rectangular prism, 10-30 $\mathrm{mm}$ high and $0.01-0.10 \mathrm{~mm}$ wide. In order to achieve a high enough radiation strength, the tiny source width is compensated by

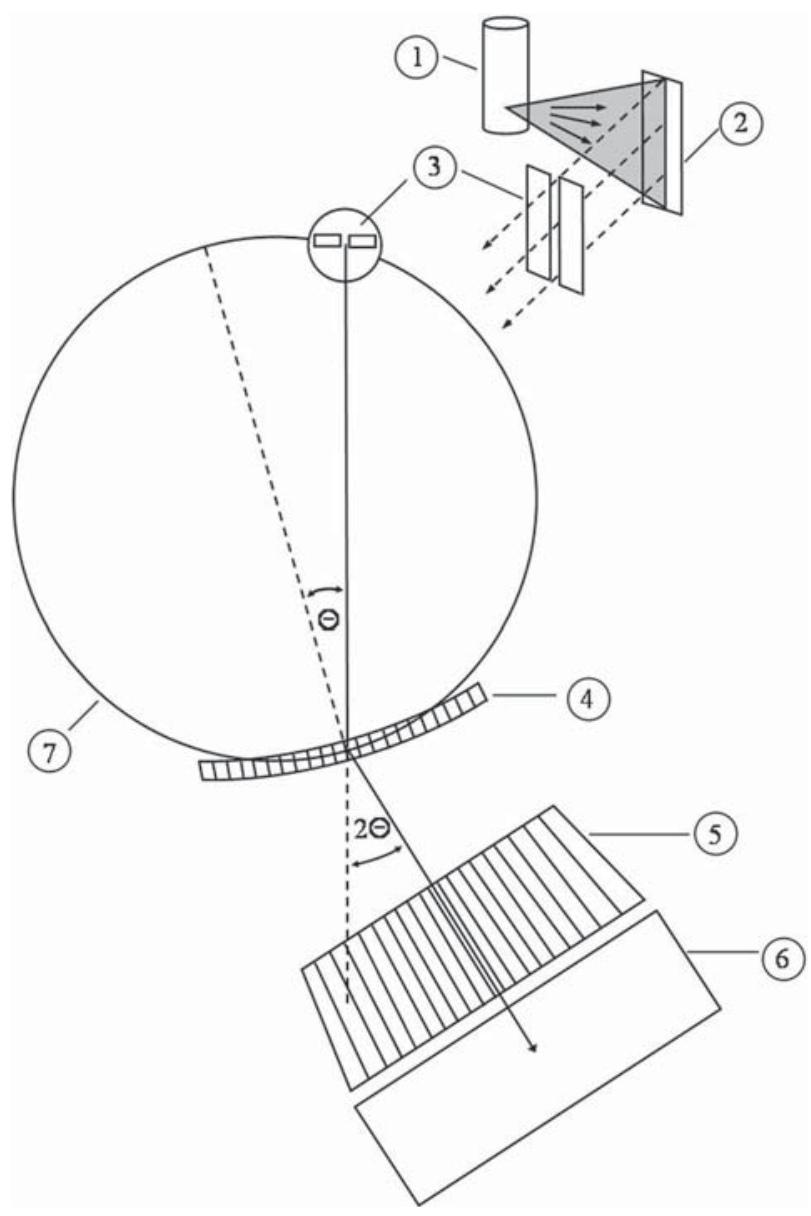

FIG. 1. Schematic drawing of the modified DuMond slit geometry: (1) X-ray tube, (2) target, (3) slit, (4) cylindrically bent crystal, (5) Soller slit collimator, (6) detector, and (7) focal circle.

a larger depth of several $\mathrm{mm}$. To preserve the resolution, the center of the prism should be located on the Rowland circle and its depth axis must be aligned on the direction defined by the source and crystal centers. For X-rays, however, this standard geometry is less appropriate because $\mathrm{X}$-rays have in general a lower energy than $\gamma$-rays and are therefore more sensitive to the self-absorption in the source. Thus, for $\mathrm{x}$-ray spectroscopy measurements the spectrometer is usually operated in the so-called modified DuMond slit geometry.

In this geometry (Fig. 1) a narrow rectangular slit is placed on the focal circle at a fixed position and serves as the effective source of radiation. The target placed behind the slit can be tilted around a vertical axis to make a certain angle with respect to the target-crystal direction. The variation of the counting rate as a function of the target alignment depends on the self-absorption of the X-rays in the target and the size of the target surface viewed by the crystal through the slit. Both quantities decrease with growing angles but with opposite effects on the counting rate. Depending on the energy of the measured X-rays, there is thus an optimum tilt angle for which the intensity of the radiation impinging on the crystal is maximum. Two further advantages are provided by this geometry: first the slit width can be adjusted to obtain the desired instrumental resolution and second the line shapes of the 


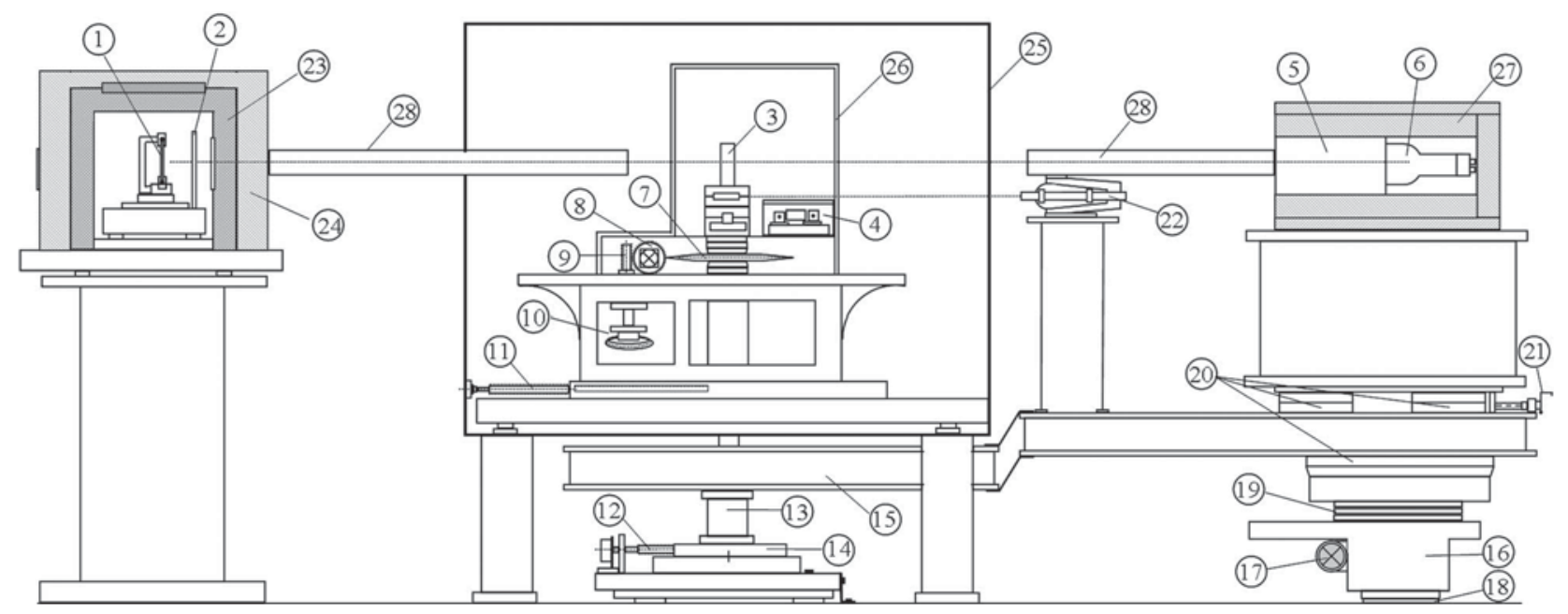

FIG. 2. Side view of the DuMond crystal spectrometer: (1) target, (2) slit, (3) crystal, (4) optical laser interferometer, (5) Soller slit-collimator, (6) detector, (7) large tooth wheel, (8) small tooth wheel and second worm gear (behind), (9) first worm gear, (10) step motor for the crystal rotation, (11) screw rod for the crystal focusing, (12) screw rod for the detector axis focusing, (13) detector rotation spindle, (14) movable table of the detector rotation spindle, (15) detector rotation arm, (16) detector automotive carriage, (17) step motor of the detector carriage, (18) circular rail, (19) large ball bearing, (20) Schneeberger minirails, (21) manual adjustment of the collimator position, (22) laser of the detector position optical tracking system, (23) target chamber, (24) lead shielding, (25) external thermostatic housing, (26) internal thermostatic housing, (27) lead shielding, and (28) evacuated pipe.

measured X-rays are no more affected by thermal deformations of the target.

A side view of the spectrometer is shown in Fig. 2. The most important parts of the instrument are the target chamber, the bent crystal, the optical laser interferometer, the collimator, and the detector. The X-rays diffracted by the crystal are recorded by a Phoswich scintillation detector. In front of the detector a Soller slit collimator is used to shield the detector from the radiation coming directly from the source. The spectra are collected by step scanning over the desired angular range. In the DuMond geometry, if the crystal revolves by an angle $\theta$, the collimator-detector system must be rotated around the same axis by $2 \theta$. Furthermore, as the Rowland circle rotates with the crystal, after a crystal rotation the source of radiation is no longer on the focal circle and the apparent width of the source becomes wider, which leads to a resolution loss of the instrument. Therefore, in DuMond spectrometers the focusing distance $f$, i.e., the distance between the crystal and the source, has to be adjusted whenever the defocusing distance given by $\delta f=R \cdot(1-\cos (\theta))$, where $R$ is the radius of curvature of the crystal, exceeds a value of the order of $\sim R / 1000$.

The Bragg angles are measured by means of an optical laser interferometer with an accuracy of approximately $0.2 \mu \mathrm{rad}$. The most temperature-sensitive elements of the spectrometer, namely, the laser interferometer and the bent crystal, are enclosed in a double thermostatic housing which keeps their temperature constant around $23{ }^{\circ} \mathrm{C}$ with a precision better than $0.05^{\circ} \mathrm{C}$. The angular range covered by the spectrometer is $\pm 20^{\circ}$, which corresponds for the first order of diffraction of a $\mathrm{SiO}_{2}(110)$ crystal to a theoretical minimum energy of about $7.4 \mathrm{keV}$.

The energy of any point $i$ of a measured angular X-ray spectrum can be calculated, using the following relation:

$$
E_{i}(\mathrm{keV})=\frac{h \cdot c}{2 \cdot d_{h k l}(\AA)} \cdot \frac{n}{\sin \left(\theta_{i}-\theta_{0}\right)} .
$$

In the above equation, $h \cdot c=12.398419(\mathrm{keV} \AA)$ is the Plank's constant multiplied by the velocity of light in vacuum, $d_{h k l}$ is the spacing constant of the crystal diffraction planes, $n$ is the diffraction order, and $\theta_{0}$ is the "spectroscopic zero position." The latter can be simply determined by measuring the same X-ray line on both sides of diffraction and by using then the relation $\theta_{0}=1 / 2\left(\theta_{+}+\theta_{-}\right)$, where $\theta_{+}$and $\theta_{-}$stand for the centroid Bragg angles corresponding to the line measured on the right (positive angles) and left (negative angles) sides of diffraction. The spacing constant $d_{h k l}$ can be determined similarly by measuring a reference X-ray line of well known energy $E_{0}$ on both sides of diffraction:

$$
d_{h k l}(\AA)=\frac{12.398419(\mathrm{keV} \cdot \AA)}{2 \cdot \sin \left(\frac{\theta_{+}-\theta_{-}}{2}\right)} \cdot \frac{n}{E_{0}(\mathrm{keV})} .
$$

Finally, the X-ray spectrum, calibrated in energy, can be analyzed by means of any least-squares-fitting program.

\section{B. Construction details}

\section{Target chamber}

The X-ray source is defined by a rectangular slit located on the focal circle of the spectrometer at a fixed position. The slit is made of two juxtaposed lead plates which are $40 \mathrm{~mm}$ high, $6 \mathrm{~mm}$ wide, and $5 \mathrm{~mm}$ thick. The distance between the two plates, i.e., the slit width, can be adjusted. If the slit width is increased, a wider part of the target surface is viewed by the crystal and the luminosity of the spectrometer is enhanced but the instrumental resolution becomes worse. Typical slit widths of $0.05-0.15 \mathrm{~mm}$ are used.

Most targets consist of $27 \mathrm{~mm}$ high $\times 4 \mathrm{~mm}$ wide metallic foils. Thicknesses vary from $\sim 10^{-3} \mathrm{~mm}$ to $\sim 10^{-1} \mathrm{~mm}$. Powder samples are prepared by dusting the powder onto a thin sticky backing having similar dimensions as the metallic foils. For gaseous samples a $3 \mathrm{~cm}$ in diameter and $3 \mathrm{~cm}$ high 
circular cell with a $11 \mathrm{mg} / \mathrm{cm}^{2}$ thick kapton wall is employed. Gas pressures up to 3 bars can be used. Other target cells with metallic or polymeric walls were developed for liquid targets such as $\mathrm{Hg} .{ }^{35}$ The distance $d$ between the slit center and the vertical axis of the solid target or the gaseous or liquid cell can be varied from $1.75 \mathrm{~cm}$ to $4.75 \mathrm{~cm}$.

The alignment and verticality of the targets can be adjusted by means of remote-controlled step motors providing the possibility to optimize the target set-up for maximum counting rates. The smallest angular steps that can be made for the source alignment and source verticality are $0.225^{\circ}$ and $0.00083^{\circ}$, respectively. The transverse position of the slit can also be adjusted by means of a remote-controlled step motor with a precision of $1.0 \mu \mathrm{m}$.

The target fluorescence is produced by irradiating the sample with the bremsstrahlung of a side-window Coolidgetype X-ray tube. Depending on the project, X-ray tubes equipped with anodes of $\mathrm{Au}$ (1 mm thick Be window), $\mathrm{Cu}$ and $\mathrm{Cr}(0.5 \mathrm{~mm}$ thick Be window), and $\mathrm{Sc}(0.3 \mathrm{~mm}$ and $0.15 \mathrm{~mm}$ thick Be windows) can be used. The bremsstrahlung and the characteristic X-ray radiation of the tube are emitted in a cone having an aperture of about $30^{\circ}$. The X-ray tube is mounted vertically on a circular port on the top of the target chamber with the side-window in the direction of the target. The X-ray tube port can be rotated around a vertical axis passing through the center of the sample for performing angular distribution measurements. The angle between the axis of the $\mathrm{X}$-ray tube beam and the target-crystal direction can be varied this way between $30^{\circ}$ and $150^{\circ}$ with angular steps of $30^{\circ}$. The water-cooled X-ray tubes are connected to a $100 \mathrm{kV} / 3 \mathrm{~kW}$ high-voltage generator.

The slit, the target, and the nose of the X-ray tube are all enclosed in a $25 \mathrm{~cm}$ long $\times 25 \mathrm{~cm}$ wide $\times 15 \mathrm{~cm}$ high vacuum chamber whose walls are made of $2 \mathrm{~cm}$ thick iron. In the center of the front wall, a circular $0.2 \mathrm{~mm}$ thick mylar window permits the exit of the fluorescence X-rays. The target chamber is pumped down to about $10^{-2}$ mbar with a rotary pump in order to minimize the oxidation of the sample, slit, and target chamber walls during irradiation. To keep the level of radiation in the lab as low as possible, the target chamber is enclosed in a $5 \mathrm{~cm}$ thick lead shielding.

\section{Crystal and crystal rotation mechanism}

The spectrometer can be equipped with different highquality single crystals such as quartz, silicon, or germanium. For the measurements presented in this work a $100 \mathrm{~mm}$ high $\times 100 \mathrm{~mm}$ wide $\times 0.5 \mathrm{~mm}$ thick quartz crystal plate was employed. The effective reflecting area was $4 \times 3 \mathrm{~cm}^{2}$. The plate was cut so that the (110) diffraction planes were normal to the front surface. A curvature radius of $315 \mathrm{~cm}$ was chosen as the best compromise between a high enough resolution and an acceptable luminosity. The instrumental resolution can be improved by increasing the curvature radius of the crystal, but the collection efficiency decreases. On the other hand the luminosity loss can be compensated by choosing a larger crystal area, but this may result in geometrical aberrations when the crystal is bent. The choice of the best crystal thickness depends on the reflectivity of the crystal and the absorption of the X-rays. The reflectivity grows with the crystal thickness as long as the latter is comparable to the extinction length. For X-rays with energies less than about $100 \mathrm{keV}$, the extinction length of quartz is of the order of several hundreds of $\mu \mathrm{m}$. Concerning the absorption, it is well known that the latter increases exponentially with the absorber thickness. Therefore, a $0.5 \mathrm{~mm}$ thick crystal plate was chosen as the best compromise.

The crystal was bent cylindrically by means of a dedicated bending device which is shown in Fig. 3. As shown, the crystal is clamped between two pairs of flat steel blocks. The two blocks of each pair are pressed together with two screws passing above, respectively, below, the crystal plate. The force which maintains the two blocks together can be adjusted with two springs. The block pairs can rotate around two vertical axes. The latter are placed symmetrically with respect to the center of the crystal and the distance between them corresponds to one fourth of the crystal length. The bending torque is applied to the crystal via two lever arms using an adjustment precision screw. The main difficulty is to apply the torque as symmetrically as possible on both sides of the crystal. Indeed, only in this case the crystal will be given the correct cylindrical shape. The two pairs of blocks are made of hardened stainless steel which is characterized by a long
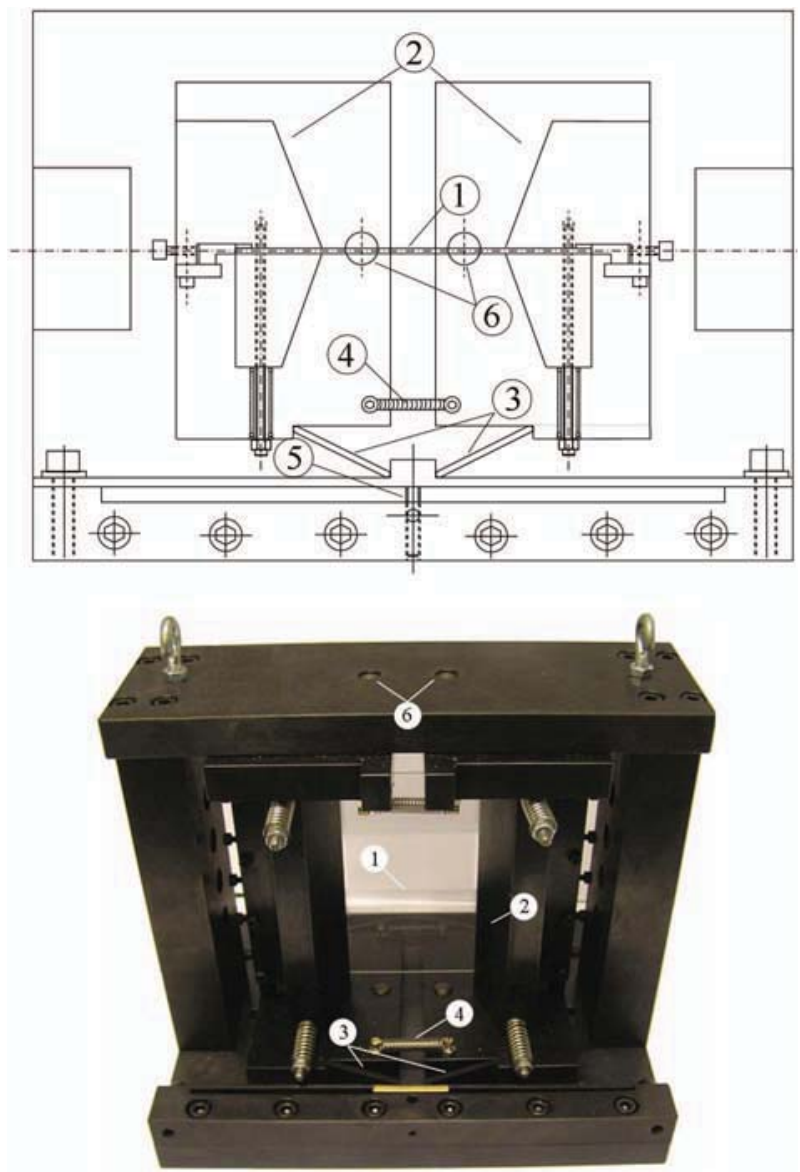

FIG. 3. Schematic diagram (top view) and photograph (view from the backside) of the bending device: (1) crystal lamina, (2) clamping blocks, (3) lever arms, (4) restoring springs, (5) bending screw, and (6) clamping block axes. 
term mechanical stability. In addition the crystal and bending device are enclosed in a thermostatic housing where the temperature fluctuations are kept below $0.05^{\circ} \mathrm{C}$.

The spacing constant $d_{110}$ of the $\mathrm{SiO}_{2}(110)$ crystal was determined using the $\mathrm{K} \alpha_{1} \mathrm{X}$-ray line of $\mathrm{Gd}$. In order to increase the precision on the $d_{110}$ constant, the $\mathrm{Gd} \mathrm{K} \alpha_{1} \mathrm{X}$ ray line was measured on both sides of diffraction in first, second, third, fourth, and fifth orders. For each diffraction order, the constant and its uncertainty were determined, using for the energy of the $\mathrm{Gd} \mathrm{K} \alpha_{1}$ transition the value of 42.99672(44) keV. ${ }^{39}$ From the five measurements the following average value was found: $d_{110}=2.456549(11) \AA$. Note that the relative error of about $4.5 \mathrm{ppm}$ originates mainly from the uncertainty on the energy of the reference line.

The crystal is rotated by means of a high-precision twostage tooth wheel mechanism. The $100 \mathrm{~mm}$ in diameter rotation axis is mounted in a ball-cage bearing. Thanks to a static compression of the balls of $5 \mu \mathrm{m}$, an accuracy of about $0.2 \mu \mathrm{m}$ is obtained for the crystal axis position. The crystal rotation is driven by a step motor and two worm gears in cascade. The big wheel fitted to the crystal axis has 360 teeth and is $440 \mathrm{~mm}$ in diameter. It is driven by the horizontal worm gear. The smaller wheel with 60 teeth is $120 \mathrm{~mm}$ in diameter and is driven by the vertical worm gear which is fixed to the step motor axis through a flexible connector to reduce the motor vibrations. One motor step corresponds to a crystal rotation of 0.075 arc sec. The big tooth wheel is made of cast iron, the two worm gears of special hardened and polished steel, and the small tooth wheel of a bronze alloy. Furthermore, in order to keep the backlash of the system at a small value, a constant restoring torque of about $2.5 \mathrm{~N}$ m provided by a counterweight of lead is applied to the big tooth wheel. The reproducibility of the rotation mechanism was probed and found to be of the order of 0.15 arc sec, whereas the observed maximal nonlinearities amount to about \pm 5 arc sec. Each crystal rotation is carried out via an acceleration-constant speed-deceleration sequence. The acceleration and deceleration ranges are 800 steps long and the maximum motor speed is 1400 steps/s, which corresponds for the crystal rotation axis to an angular velocity of $1.75^{\circ} / \mathrm{min}$. This mode of operation of the step motor permits a smoother and therefore more accurate rotation of the crystal.

\section{Optical laser interferometer}

The Bragg angles are measured by means of an optical laser interferometer with a precision of several milli-arcsec. The schematic layout of the interferometer is represented in Fig. 4. A dedicated He-Ne laser emits two beams of red light with slightly different optical frequencies, $f_{1}$ and $f_{2}$. The frequency difference $f_{1}-f_{2}$ of about $2 \mathrm{MHz}$ is very stable and represents the reference signal of the interferometer. Both beams are polarized linearly, the first one in the horizontal plane, the second one in the vertical plane, so that the two signals can be separated by optical elements sensitive to the direction of polarization. The double beam is directed to the beam splitter which separates the two components according

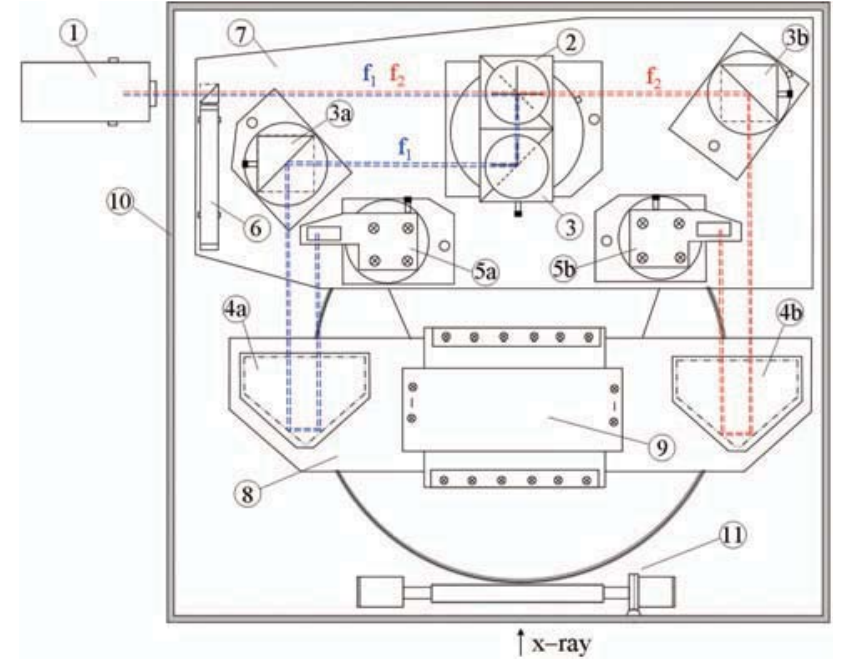

FIG. 4. Optical laser interferometer (top view): (1) two-frequency laser, (2) beam splitter, (3, 3a, 3b) plane mirrors, (4a, 4b) cube corner reflectors, $(5 \mathrm{a}, 5 \mathrm{~b})$ roof prisms (end reflectors), (6) photodetector, (7) fixed table, (8) rotary table, (9) crystal bending device, (10) thermostatic box, and (11) driving system of the crystal.

to their polarization. The beam $f_{1}$, deflected by the mirrors and by the cube corner reflector, reaches the roof prism (end reflector). The latter reflects the beam which is redirected to the beam splitter by the same optical elements but along a lower lying path. In the beam splitter the beam $f_{1}$ is recombined with the beam $f_{2}$ which has followed a similar path on the other side of the interferometer. The two beams interfere then in the photodetector which provides an electric signal whose frequency corresponds to the beat frequency of the two beams.

The crystal and all optical elements of the interferometer, except the laser, are enclosed in a thermostatic box where the temperature is kept constant within $0.05^{\circ} \mathrm{C}$. The box contains two tables. A fixed one, which is used as a support for all optical elements, except the cube corner reflectors, and a rotary one, fixed to the crystal rotation axis, which supports the crystal bending device and the two cube corner prisms. When the rotary table is not moving, the frequency of the beat signal provided by the photodetector is the same as the reference frequency given by the laser so that the angle measured by the system remains unchanged. However, as soon as the table is rotated, due to the displacement of the cube corner reflectors which are moving in opposite directions, the two frequencies $f_{1}$ and $f_{2}$ are Doppler shifted, one frequency being increased, the other one decreased, and the beat frequency provided by the detector is no longer equal to the reference frequency. The controller receives the reference and measured signals, processes them, and determines the actual crystal angle in optical units. One optical unit corresponds to a difference of $\lambda / 40$ between the optical paths of the two beams. More detailed descriptions of similar interferometers including some theoretical aspects can be found in Refs. 15 and 36.

The interferometer was calibrated, using a Moore rotary table $^{37}$ and a Hilger-Watts TA53 autocollimator. The combination of these two instruments permitted us to determine 241 
crystal angles between $-20^{\circ}$ and $+20^{\circ}$ with an angular step of $600 \mathrm{arc} \mathrm{sec}$ and a precision of $0.2 \mathrm{arc} \mathrm{sec}$.

The method consisting of measuring the optical path difference between the two beams via the Doppler effect presents the great advantage to be less sensitive to the frequency instability of the laser because both light beams are affected in the same way. However, as the measured displacements of the cube corners during a rotation are determined through the frequency changes of the two beams but are expressed in units of $\lambda / 40$, the light velocity inside the thermostatic housing must be known. For this reason, the temperature, pressure, and humidity of air inside the thermostatic box are measured by the system. From the obtained values the controller computes the so-called compensation factor (ratio between the light velocity in the thermostatic house and in vacuum) and multiplies then the interferometer value by the calculated compensation factor.

\section{Collimator and collimator rotation mechanism}

The Soller slit collimator installed in front of the detector serves mainly to suppress the direct photon beam which is spatially overlapping with the diffracted beam at small Bragg angles. In addition the collimator diminishes the background originating from the elastic and inelastic scattering of the incoming photon beam in the crystal.

The collimator consists of 24 parallel rectangular slits which are $660 \mathrm{~mm}$ long, $110 \mathrm{~mm}$ high, and $2 \mathrm{~mm}$ wide. It was constructed by inserting 23 stainless steel plates in a hollow prismatic frame made of cast iron. The side walls of the frame are rectangular $(660 \mathrm{~mm}$ long $\times 105 \mathrm{~mm}$ high $\times 20 \mathrm{~mm}$ thick), whereas the bottom and top walls are trapezoidal with the same length and thickness as the side walls but a width of $134 \mathrm{~mm}$ on the rear side and of $123.95 \mathrm{~mm}$ on the front side. The 23 rectangular plates have the same length and height as the side walls of the frame but a thickness varying linearly from $2.00 \mathrm{~mm}$ on the rear side to $1.56 \mathrm{~mm}$ on the front side. With this design, the transmission of the collimator amounts to $51 \%$. The transmission curve of the collimator has a nearly trapezoidal shape with angular widths of about 200 arc sec for the plateau and 1100 arc sec for each flank.

The collimator and the detector are supported by a carriage that can move autonomously on a circular rail made of hardened stainless steel. The radius of the circular rail is $2750 \mathrm{~mm}$ and its total length $4743 \mathrm{~mm}$, which allows to cover the range of $\pm 40^{\circ}$ of the collimator-detector system. During the acquisition of an X-ray spectrum the collimator-detector system is automatically aligned to the double Bragg angle $2 \theta_{B}$ thanks to an optical tracking device consisting of a laser located on the collimator arm, a mirror placed on the crystal axis, and a fixed two-segment photodiode. The error signal provided by the photodiode serves to control the step motor of the collimator-detector carriage. The accuracy of this optical feedback system was probed over the entire angular range of the detector and found to be better than about $25 \%$ of the collimator plateau width.

\section{Detectors}

For the detection of the diffracted X-rays a Phoswich scintillation counter with a $25.4 \mu \mathrm{m}$ thick aluminum entrance window is usually employed. This $12.7 \mathrm{~cm}$ in diameter detector consists of a front $0.635 \mathrm{~cm}$ thick $\mathrm{NaI}(\mathrm{Tl})$ crystal followed by an optically coupled $5.08 \mathrm{~cm}$ thick rear CsI(Tl)) crystal, both mounted on the same photomultiplier tube. The first crystal serves to detect the radiation of interest, whereas the second crystal acts as an active shielding permitting to reject events corresponding to background and higher diffraction order photons. Thanks to the different decay times characterizing the two crystals, the fluorescence signals corresponding to the two scintillators can indeed be distinguished and separated by a dedicated hardware using a pulse shape analysis module. Three categories of events are recognized by the electronics: events corresponding to a single signal from the $\mathrm{NaI}(\mathrm{TI})$ crystal, to a single signal from the CsI(TI) crystal or to simultaneous signals from both scintillators. Good events are those belonging to the first category since they correspond to low energy photons entering the detector from the proper side and with the right direction defined by the collimator angle $2 \theta_{B}$. Events belonging to the second category are mainly due to cosmic rays, those belonging to the third one to higher energy photons that have made a Compton interaction in the first crystal and a Compton or photoeffect in the second one. Events from the second and third categories are rejected by the detection electronics.

The two crystals and the photomultiplier tube are contained in a hermetically sealed $0.635 \mathrm{~cm}$ thick stainless steel housing. Furthermore, in order to reduce the background the detector is placed in a thick $\mathrm{Pb}-\mathrm{Cu}-\mathrm{Al}$ shielding. The relative energy resolution of the Phoswich detector varies as a function of the photon energy between $20 \%$ and $30 \%$.

For applications for which a better energy resolution is needed the Phoswich scintillator is replaced by a semiconductor detector. Two such semiconductor detectors are available. The first one is a $20 \mathrm{~cm}^{3}$ intrinsic germanium LeGe detector having a thickness of $1 \mathrm{~cm}$. Thanks to its big surface which permits to cover about $85 \%$ of the diffracted photon beam this detector can be used for measurements that need a good detection efficiency with a high enough spectrometer luminosity. The second one is a $0.4 \mathrm{~cm}^{3}$ lithium-doped silicon $\mathrm{Si}(\mathrm{Li})$ detector having a thickness of $0.5 \mathrm{~cm}$. It is mainly employed when the diffracted photon beam is intense and its energy below about $20 \mathrm{keV}$.

\section{Electronics and data acquisition}

The schematic block diagram of the driving and acquisition electronics is shown in Fig. 5. The spectrometer is equipped with four remote-controlled step motors which serve to rotate the crystal (motor labeled CRY in Fig. 5) and the collimator-detector system (COL) as well as to translate the crystal (FOC) and detector (FOP) spindles when the focal distance of the spectrometer has to be adjusted. Three further remote-controlled step motors are used to optimize the alignment (SAL) and verticality (SVE) of the target and to align the transverse position of the slit (SLT) onto the 


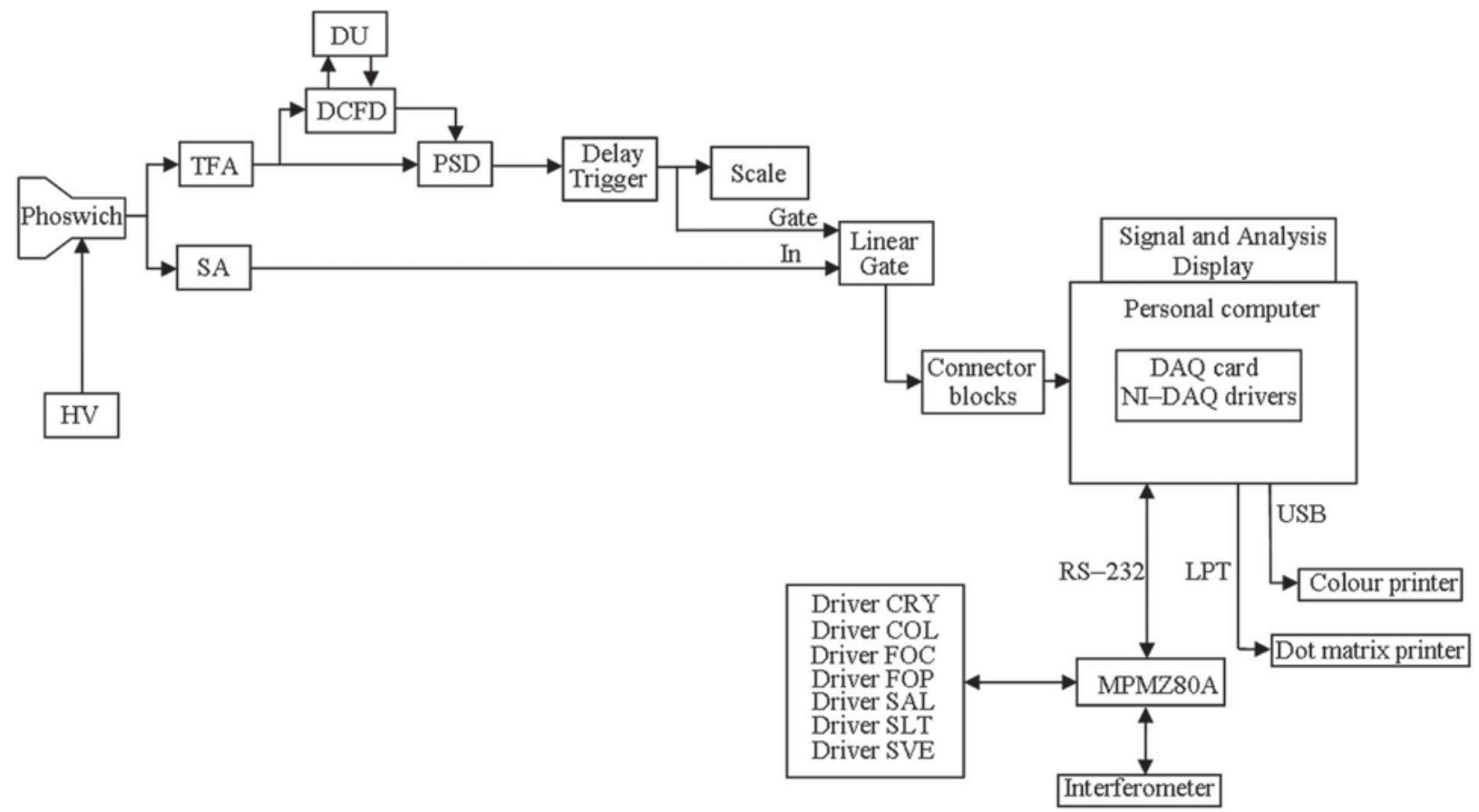

FIG. 5. Block diagram of the driving and acquisition electronics of the spectrometer. HV stands for the high-voltage applied to the Phoswich detector (HV $=$ $1280 \mathrm{~V}$ in our case), SA for the spectroscopy amplifier (Canberra, model 2021), TFA for the timing filter amplifier (Ortec, model 454), DCFD for the differential constant fraction discriminator (Canberra, model 2129), DU for the delay unit (home made module), and PSD for the pulse shape discriminator (Canberra, model 2160A).

target-crystal direction. The steering of the spectrometer and target chamber step motors is controlled by a Z80 microprocessor (MPMZ80) via VME (Virtual Machine Environment) micro-step generators and linear amplifiers. The communication between the PC and the VME modules is performed through a RS-232 connection at a baud rate of 9600 bytes/s. The controller of the laser interferometer is also connected to the Z80 microprocessor through a GPIB (General Purpose Interface Bus) interface.

The main function of the acquisition electronics is to amplify, shape, and sort the signals provided by the Phoswich detector. The Phoswich photomultiplier (PM) yields two output signals: the first one provided by the PM anode is used to determine the total energy deposed in the detector (energy signal), and the second one, which is delivered by the last PM dynode and is thus faster is used for timing purposes (timing signal). The energy signal is sent to the input of a spectroscopy amplifier (SA) whose gain can be chosen and the shaping time is kept fixed at $1 \mu \mathrm{s}$. The timing signal is sent through a timing filter amplifier (TFA) to the pulse shape discriminator (PSD). The latter permits to separate the X-ray photons that lose their energy entirely in the main crystal from those that lose their energy, partly or totally, in the second crystal. The separation of the two signals is possible because of their different rise time. The PSD module provides a gate signal if the timing signal corresponds to a good event and an anti-gate signal in the opposite case. The gate or anti-gate signal and the signal from the spectroscopy amplifier are both sent to a linear gate. If the gate signal is present, the linear gate forwards the energy signal to a multifunction data acquisition card (DAQ) which is mounted in the computer itself. If it is an anti-gate signal, the energy signal is ignored by the linear gate.
The DAQ card converts the analog energy signal into a digital one, which can be then handled with the appropriate software.

The computer is equipped with a PCI-6040 E series Multifunctional I/O card made by National Instruments. ${ }^{38}$ This card consists of an analog to digital converter (ADC) with 8 input channels for voltage measurements, a digital to analog converter (DAC) with 2 output channels for generating voltages, several counters and digital input/output (I/O) ports. The precision of the conversion of the analog input signal into a digital signal depends on the number of bits used by the ADC. The DAQ card installed in our computer has a resolution of 12 bits (4096 channels), a $250 \mathrm{kB} / \mathrm{s}$ multichannel sampling rate and a dynamical range of $0-10 \mathrm{~V}$.

The data acquisition and the spectrometer operation are controlled by a self-written program using the LabVIEW 8.0 software developed by National Instruments. The main input parameters of the program controlling the automatic data acquisition are the start position of the crystal angular scan, the crystal focal distance, the number of steps, and the step length needed to cover the angular range to be measured, the acquisition time per point which is chosen depending on the count rate, the energy window which allows to reject higher reflection order and background events, and the number of interferometer's readings per point. The angular spectrum is built point by point, saved, and displayed graphically on the screen.

\section{OPERATIONAL CHARACTERISTICS}

\section{A. Spectrometer resolution}

The instrumental resolution of the spectrometer depends mainly on the slit width, crystal mosaicity, and precision of 


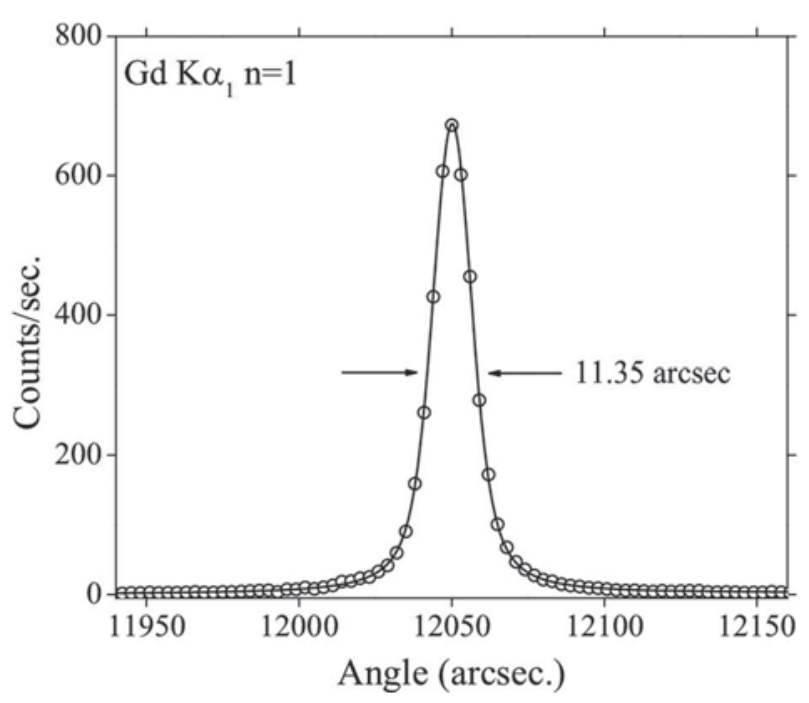

FIG. 6. The Gd K $\alpha_{1}$ X-ray emission line measured in first order of diffraction. The experimental points (open circles) were fitted with a Voigt function and a linear background. The solid line represents the total fit.

the crystal curvature. The angular instrumental resolution was determined using the $\mathrm{K} \alpha_{1}$ X-ray emission line of a $36 \mathrm{mg} / \mathrm{cm}^{2}$ thick metallic foil of $\mathrm{Gd}$ measured in first order of diffraction. The sample was irradiated with the bremsstrahlung produced by a $\mathrm{Au}$ anode $\mathrm{x}$-ray tube which was operated at $80 \mathrm{kV}$ and $30 \mathrm{~mA}$. A slit width of $0.10 \mathrm{~mm}$ was used. The angular spectrum (Fig. 6) was analyzed with the PeakFit ${ }^{40}$ program, using a Voigt function to fit the $\mathrm{K} \alpha_{1} \mathrm{X}$-ray line. A Voigt function was chosen because the latter corresponds to the convolution of the natural Lorentzian shape of an X-ray transition with the Gaussian instrumental response of the spectrometer. In the fitting procedure the intensity, peak position, and Gaussian standard deviation as well as a linear background were let free while the value of the Lorentzian width derived from the tables of Campbell and Papp ${ }^{41}$ was kept fixed. From the analysis the value of $\sigma_{\theta}=4.82 \pm 0.04 \mathrm{arc} \mathrm{sec}=23.4 \pm 0.2 \mu \mathrm{rad}$ was obtained for the standard deviation of the Gaussian function. The corresponding full width at half maximum (FWHM) angular instrumental resolution is thus $\Gamma_{\theta}=2 \cdot \sqrt{2 \ln 2} \cdot \sigma_{\theta}$ $=11.4(1)$ arc sec $=55.0(9) \mu \mathrm{rad}$. The energy instrumental resolution $\sigma_{E}$ is related to $\sigma_{\theta}$ (expressed in rad) by the following equation:

$$
\sigma_{E}=E \cdot \operatorname{ctg}\left(\theta_{B}\right) \cdot \sigma_{\theta},
$$

where $E$ is the energy of the observed X-ray line and $\theta_{B}$ is the Bragg angle of the latter. Using this equation an energy resolution $\sigma_{E}=17.1 \mathrm{eV}$ is found for the $\mathrm{Gd} \mathrm{K} \alpha_{1} \mathrm{X}$-ray line whose energy is $42.996 \mathrm{keV}$, whereas for the Mo $\mathrm{K} \alpha_{1}$ $\mathrm{X}$-ray line which has an energy of $17.479 \mathrm{keV}$ a value of $2.8 \mathrm{eV}$ is obtained. This illustrates the fact that for crystal spectrometers the energy resolution becomes poorer with increasing photon energy $E$, the resolution deterioration being nearly proportional to $E^{2}$.

In the DuMond slit-geometry the effective size of the $\mathrm{X}$-ray source is defined by the slit width. The latter influences the instrumental resolution as well as the measured intensity since the solid angle of the spectrometer is directly propor-

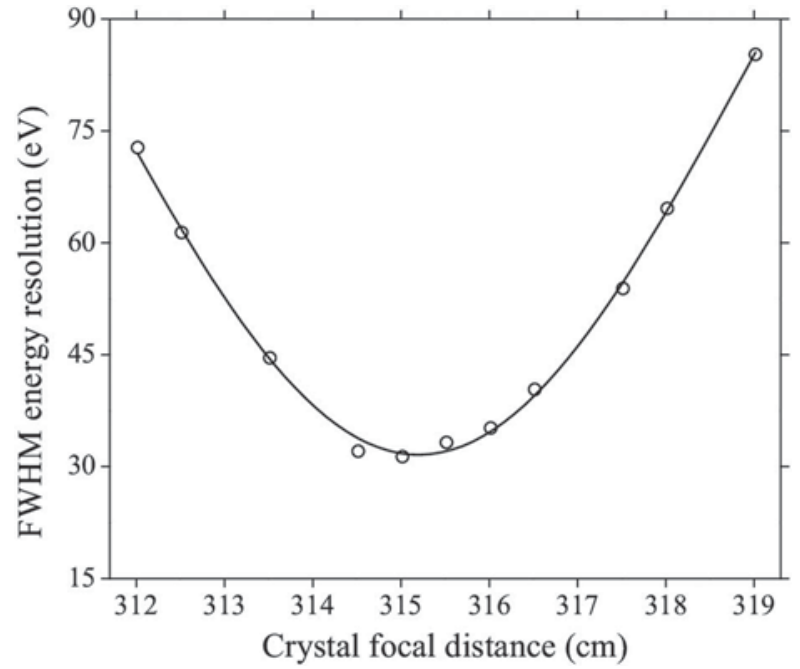

FIG. 7. Variation of the energy resolution of the $\mathrm{Gd} \mathrm{K} \alpha_{1} \mathrm{X}$-ray line as a function of the focal distance of the crystal. The solid line represents the 2 nd degree polynomial used to interpolate the experimental points (open circles).

tional to the employed slit width. To demonstrate the effect of the source size on the instrumental resolution, the Mo K $\alpha_{1}$ $\mathrm{X}$-ray emission line was measured in 1 st order of diffraction with different slit widths. From the results provided by the fitting procedure it was found that $\sigma_{E}$ increases as a function of the slit width, varying from $2.40 \mathrm{eV}$ to $3.11 \mathrm{eV}$ when the slit width grows from $0.05 \mathrm{~mm}$ to $0.15 \mathrm{~mm}$.

In order to probe the effect of the focal distance $f$ (see Sec. II A) on the instrumental resolution, the $\mathrm{Gd} \mathrm{K} \alpha_{1} \mathrm{X}$-ray emission line was measured in first order of diffraction at different slit-to-crystal distances. The results of these measurements are presented in Fig. 7. As shown the best energy resolution is found for a focal distance of $315.2 \mathrm{~cm}$. The latter corresponds to a radius of curvature $R$ of $315.7 \mathrm{~cm}$, a value which is consistent within the experimental uncertainties with the nominal value of $R(315 \mathrm{~cm})$. The instrumental resolution can also be improved by measuring the X-ray spectra at higher diffraction orders. To demonstrate this effect, the $\mathrm{Gd} \mathrm{K} \alpha_{1}$ $\mathrm{X}$-ray line was observed at five different orders of diffraction. From Fig. 8 one can see that the instrumental resolution between the 1 st and the 5 th order is improved by a factor of about 5. However, as shown the spectrometer efficiency decreases also unfortunately with the diffraction order $n$.

\section{B. Spectrometer sensitivity}

The DuMond curved crystal spectrometer of Fribourg is characterized by good peak-to-background ratios and thus by a high sensitivity. To illustrate the sensitivity of the instrument the extremely weak $\mathrm{Gd} \mathrm{K} \alpha_{3} \mathrm{X}$-ray emission line corresponding to the dipole-forbidden $2 s_{1 / 2} \rightarrow 1 s_{1 / 2}$ transition was measured. The measurements were performed with the same experimental set-up as the one used in the spectrometer resolution measurements.

As mentioned before, the best angular resolution is obtained when the slit-to-crystal distance is adjusted for each measured X-ray line. However, when the measured x-ray spectrum extends over a large angular range with many lines 


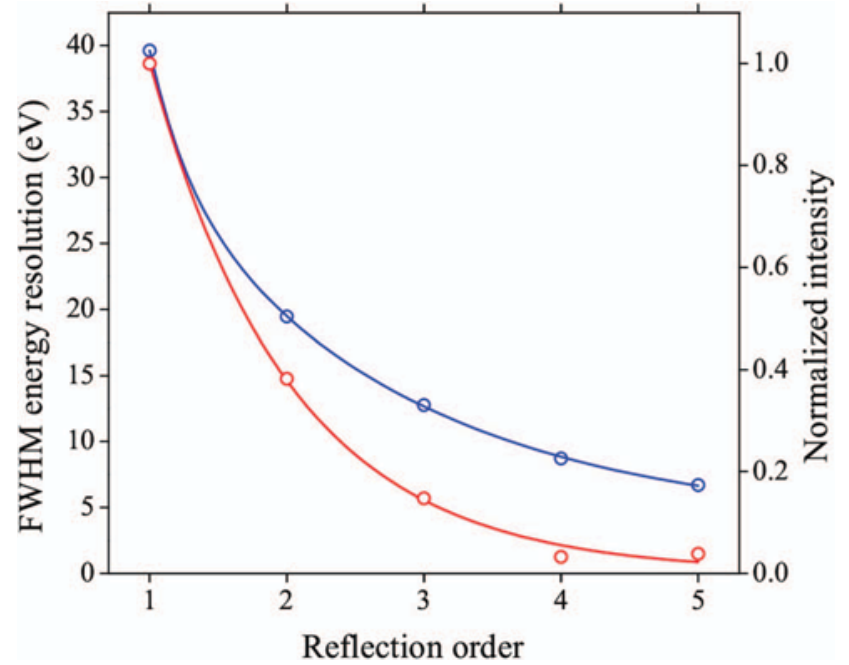

FIG. 8. Variation of the energy resolution (blue open circles, upper curve) and relative intensity (red open circles, lower curve) of the $\mathrm{Gd} \mathrm{K} \alpha_{1} \mathrm{X}$-ray line as a function of the diffraction order. The solid lines serve only to guide the eye.

or if the spectrum contains a very weak line it is more practical to measure the X-ray spectrum with an average value of the focusing distance or, in the case of a weak line, with the focusing distance corresponding to the nearest strong line. For the $\mathrm{K} \alpha_{3}$ transition the measurements were performed with the focusing distance corresponding to the $\mathrm{Gd} \mathrm{K} \alpha_{2}$ transition.

Because for measurements performed with the DuMond spectrometer the X-ray spectra are measured point by point, the time needed to measure a whole spectrum containing a weak line can be very long if the same collecting time per point is employed for all points. For this reason, the measurements were performed in two steps. First the $\mathrm{K} \alpha_{1,2}$ spectrum was measured on both sides of reflection in order to determine the zero Bragg angle required for the energy calibration of the spectrometer. In the second step the angular range corresponding to the energy region of the $\mathrm{K} \alpha_{3}$ transition was measured on the right side of reflection in 28 successive step by step scans. Each scan contained 200 equidistant points and the acquisition time per point was 200 s. Furthermore, in order to control the instrumental reproducibility and the stability of the X-ray tube intensity a short measurement of the $\mathrm{K} \alpha_{2}$ $\mathrm{X}$-ray line was performed between each fourth scan. The analysis showed, however, that the fluctuations of the intensity of the $\mathrm{K} \alpha_{2}$ line were smaller than $1.4 \%$ and the instabilities of the line position less than $0.8 \mathrm{eV}$. For this reason, no off-line corrections of the data were performed and the recorded scans were simply added together.

The measured $\mathrm{Gd} \mathrm{K} \alpha$ spectrum is presented in Fig. 9. As shown, in the energy region corresponding to the $\mathrm{K} \alpha_{3}$ line, the main contribution to the measured intensity originates from the low energy tail of the $\mathrm{K} \alpha_{2}$ line and the linear background. Some additional contribution is due to the low energy tail of the $\mathrm{K} \alpha_{1}$ line and only a very tiny part comes from the forbidden $\mathrm{K} \alpha_{3}$ transition. Therefore, to fit properly the $\mathrm{K} \alpha_{3}$ x-ray line a two step procedure was employed. In the first step, the $\mathrm{K} \alpha_{1}$ and $\mathrm{K} \alpha_{2}$ lines were fitted in order to determine the intensity of the tails in the region corresponding to the $\mathrm{K} \alpha_{3}$ tran-

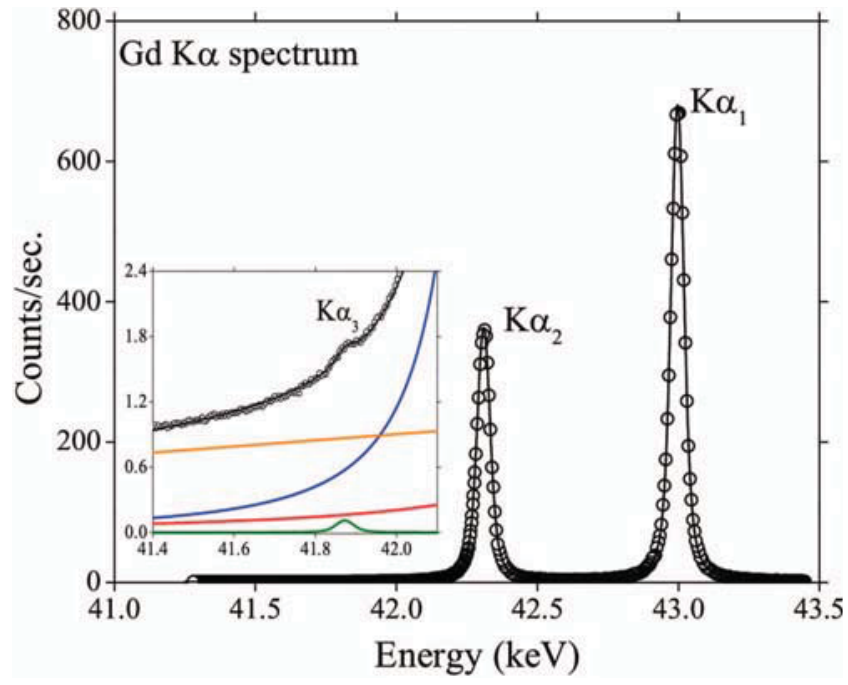

FIG. 9. The K $\alpha$ X-ray spectrum of Gd. The dots represent the experimental data and the black solid line represents the total fit. The inset shows the enlarged energy region of the $\mathrm{K} \alpha_{3}$ transition with the components used in the fit, viz (from the top to the bottom): tail of the $\mathrm{K} \alpha_{2}$ line (blue curve), linear background (orange straight line), tail of the $\mathrm{K} \alpha_{1}$ line (red curve), and $\mathrm{K} \alpha_{3}$ line (green curve).

sition. In the fitting procedure the intensities, energies, and Gaussian widths of the X-ray lines were let free, whereas the Lorentzian widths were kept fixed at the values derived from the atomic level widths quoted in the tables of Campbell and Papp. ${ }^{41}$ Then, the fitted low energy tails of the $\mathrm{K} \alpha_{1}$ and $\mathrm{K} \alpha_{2}$ lines and the linear background were subtracted from the measured total spectrum. In the second step, the residual spectrum was analyzed. Due to the extremely poor intensity of the $\mathrm{K} \alpha_{3}$ $\mathrm{X}$-ray line, in the fitting procedure only the energy and the intensity were let free, the Gaussian and Lorentzian widths of the weak transition being kept fixed. The Gaussian width was calculated from the known angular instrumental resolution while the Lorentzian width was deduced from Ref. 41.

For the energy of the $\mathrm{K} \alpha_{3} \mathrm{X}$-ray transition, a result of $41870 \pm 2 \mathrm{eV}$ was obtained. The latter was found to be consistent, within the experimental uncertainties, with another existing experimental value reported in Ref. 42 and with the theoretical prediction given in Ref. 39. Concerning the relative intensity of the forbidden transition, a ratio $I\left(\mathrm{~K} \alpha_{3}\right) / I\left(\mathrm{~K} \alpha_{1}\right)$ of $(1.61 \pm 0.11) \times 10^{-4}$ was found. This result is in good agreement with the experimental value of $(1.5 \pm 0.1) \times 10^{-4}$ quoted in Ref. 42 as well as with a theoretical prediction based on Scofield's calculations ${ }^{43}$ which gives a value of 1.7 $\times 10^{-4}$.

\section{Spectrometer precision}

\section{Probing the Ritz combination principle}

The $\mathrm{K} \alpha_{2}\left(K-L_{2}\right.$ transition), $\mathrm{K} \beta_{1}\left(K-M_{3}\right.$ transition), and $\mathrm{L} \beta_{17}\left(L_{2}-M_{3}\right.$ transition) $\mathrm{X}$-ray lines of Au were measured to check the precision of the energies determined with the spectrometer. The aim of these measurements was to probe the Ritz combination principle. According to the latter, the sum of the energies of the $K-L_{2}$ and $L_{2}-M_{3}$ transitions should be 
indeed equal to the energy of the $K-M_{3}$ transition. These measurements were experimentally challenging because the energy domain corresponding to the three transitions extended over about $70 \mathrm{keV}$ and the energy of the $L_{2}-M_{3}$ transition $(10.6 \mathrm{keV})$ was far below the lowest energy that can be usually measured with Laue type crystal spectrometers. Nevertheless, thanks to the wide angular range covered by the DuMond spectrometer of Fribourg and the thinness of the employed quartz crystal, the project could be carried out successfully and the Ritz principle verified within a relative precision of about $10^{-5}$.

In these measurements the $\mathrm{Au}$ anode of the X-ray tube itself served as source of radiation. The X-ray tube was operated at $90 \mathrm{kV}$ and $30 \mathrm{~mA}$ and it was positioned so that the axis of the emitted radiation was colinear with the target-crystal direction. The $\mathrm{K} \alpha_{2}$ and $\mathrm{K} \beta_{1} \mathrm{X}$-ray lines were observed on both sides of diffraction in order to determine for each of them the zero position of the Bragg angle scale required for the energy calibration of the spectrometer. The measured $\mathrm{K} \alpha_{2}$ and $\mathrm{K} \beta_{1}$ spectra contained 100 and 150 equidistant points, respectively. For the $\mathrm{K} \alpha_{2}$ line, the acquisition time per point was $30 \mathrm{~s}$, whereas for the $\mathrm{K} \beta_{1}$ line a time of $250 \mathrm{~s}$ was chosen. Because the $\mathrm{L} \beta_{17}$ is very weak, the zero Bragg angle was determined using the $\mathrm{L} \beta_{1} \mathrm{X}$-ray line $\left(L_{2}-M_{4}\right.$ transition). The angular range corresponding to the energy region of the $\mathrm{L} \beta_{17}$ line was measured in several successive step by step scans. Each scan contained 140 equidistant points and the total acquisition time per point was $2300 \mathrm{~s}$. The stability of the experimental setup was checked periodically by inserting short measurements of the $\mathrm{L} \beta_{1} \mathrm{X}$-ray line between each scan.

The energy spectra were fitted with Voigt functions. In the fitting procedure the Lorentzian widths, derived from Ref. 41 and the known instrumental Gaussian widths were kept fixed, whereas the intensities, centroid energies, and parameters of the linear background were let free. In order to fit properly the weak $L_{2}-M_{3}$ quadrupole transition two Voigtians had to be used so that the energy of this transition was determined from the weighted average of the energies of the two components. The fitted spectra are presented in Fig. 10, whereas the sum of the energies of the two cascading transitions is compared to the energy of the crossover transition in Table I.

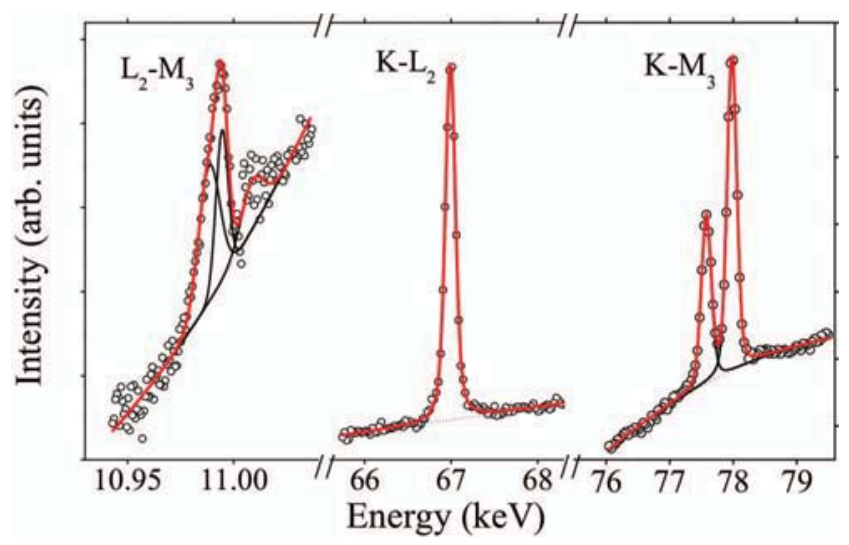

FIG. 10. Measured $L_{2}-M_{3}, K-L_{2}$, and $K-M_{2,3}$ transitions of gold (dots). The red solid lines show the total fits to the experimental data points.
TABLE I. Comparison between the sum of the energies of the $K-L_{2}$ and $L_{2}-$ $M_{3}$ cascading transitions and the energy of the crossover $K-M_{3}$ transition.

\begin{tabular}{lccc}
\hline \hline & $\begin{array}{c}K L_{2}+L_{2} M_{3} \\
(\mathrm{eV})\end{array}$ & $\begin{array}{c}K M_{3} \\
(\mathrm{eV})\end{array}$ & $\begin{array}{l}{\left[K M_{3}-\left(K L_{2}\right.\right.} \\
\left.\left.+L_{2} M_{3}\right)\right](\mathrm{eV})\end{array}$ \\
\hline This work & $77981.04 \pm 0.39$ & $77979.92 \pm 0.52$ & $-1.12 \pm 0.65$ \\
Ref. 37 & $77982.27 \pm 0.75$ & $77979.80 \pm 0.38$ & $-2.47 \pm 0.84$ \\
\hline \hline
\end{tabular}

As it can be seen from Table I, our measurements are consistent with the Ritz principle within $2 \sigma$ errors, a small but significant deviation being, however, observed if $1 \sigma$ errors are considered. The origin of the discrepancy can be explained by the energy of the $L_{2}-M_{3}$ transition which seems to be somewhat too high. Actually, the shape of the $L_{2}-M_{3}$ transition evinces some asymmetry so that it was not possible to fit it with a single Voigtian. In our opinion, this asymmetry might indicate that the $L_{2}-M_{3} \mathrm{X}$-ray line is overlapping with its unresolved $N$ x-ray satellites which originate from $L_{1} L_{2} N$ Coster-Kronig transitions. Note that the energy difference deduced from Ref. 39 is neither consistent with zero, the deviation being even more than two times bigger than ours.

\section{Precision of the interferometer}

If an X-ray line of unknown energy $E_{i}$ and a reference X-ray line of known energy $E_{0}$ are both measured at positive and negative Bragg angles, in the $n_{i}$ th and $n_{0}$ th diffraction order, respectively, the energy of the first transition can be directly deduced from the numbers $X$ in optical units (OU) provided by the laser interferometer, using the following formula: ${ }^{15}$

$$
\frac{E_{i}}{E_{0}}=\frac{n_{i}}{n_{0}} \cdot \frac{X_{n_{0+}}-X_{n_{0-}}}{X_{n_{i+}}-X_{n_{i-}}}=\frac{n_{i}}{n_{0}} \cdot \frac{X_{B}{ }^{0}}{X_{B}{ }^{i}},
$$

where $X_{n_{0+}}, X_{n_{0-}}, X_{n_{i+}}$, and $X_{n_{i-}}$ stand for the fitted centroids in OU of the reference transition and transition of interest on the positive and negative diffraction sides. The precision of the interferometer interpolation polynomial can therefore be probed by comparing the energies of an X-ray transition obtained with the standard method, i.e., the method based on Eqs. (1) and (2) and the method, based on Eq. (4), which is independent from the interferometer calibration polynomial. For this test of the interferometer precision, a new series of measurements of the $\mathrm{Gd} \mathrm{K} \alpha_{1}$ x-ray line was undertaken. The transition was measured at five different orders of diffraction on both sides of diffraction. As a reference, the $\mathrm{K} \alpha_{1} \mathrm{x}$-ray line of $\mathrm{Au}$ was chosen. The latter was measured in the 4th order on both sides of diffraction and its energy was taken from Ref. 44. To make the two methods consistent, the spacing constant $d_{110}$ of the crystal was also determined with the $\mathrm{Au} \mathrm{K} \alpha_{1}$ transition. A slightly bigger value than the one obtained from the first series of measurements of the Gd K $\alpha_{1}$ transition (see Sec. II B 2) was found, the two values being, however, consistent within the combined errors. The energies of the $\mathrm{Gd}$ $\mathrm{K} \alpha_{1} \mathrm{x}$-ray emission line obtained from the first method using the Au-based $d_{110}$ constant are shown in the second row of Table II as a function of the five diffraction orders. An average energy of $42.99648 \mathrm{keV}$ with a standard deviation of 
TABLE II. Variation of the energy of the Gd K $\alpha_{1}$ X-ray line as a function of the diffraction order.

\begin{tabular}{lllll}
\hline \hline Order & 1 & 2 & 3 & 5 \\
\hline Energy $^{\mathrm{a}}(\mathrm{keV})$ & $42.99623(7)$ & $42.99587(5)$ & $42.99668(7)$ & $42.99672(8)$ \\
Energy $^{\mathrm{b}}(\mathrm{keV})$ & $42.99609(19)$ & $42.99581(18)$ & $42.99674(18)$ & $42.99690(2)$ \\
\hline \hline
\end{tabular}

${ }^{a}$ Values determined from Eqs. (1) and (2)

${ }^{b}$ Values determined from Eq. (4).

$0.00038 \mathrm{keV}$ is found. The energies deduced from the second method (Eq. (4)) are presented in the third row of Table II. In this case the average energy of the $\mathrm{Gd} \mathrm{K} \alpha_{1}$ $\mathrm{x}$-ray line is $42.99660 \mathrm{keV}$ with a standard deviation of $0.00058 \mathrm{keV}$. From the comparison of the values quoted in the second and third rows of Table II, one can conclude that the energies obtained with the two methods are consistent within the quoted uncertainties, except for the 5th order for which a small discrepancy is observed. It can be further noted that the two average energies are also in good agreement with the value of 42.99672(44) keV reported by Deslattes et al. ${ }^{39}$

\section{Spectrometer versatility}

X-ray Absorption Spectroscopy (XAS) measurements represent a powerful tool to characterize the electronic structure and the atomic environment (number and species of neighbor atoms) of solid, liquid, and gaseous samples. Different techniques such as EXAFS (Extended X-ray Absorption Fine Structure) and XANES (X-ray Absorption Near Edge Structure) have been developed and represent now routine works at synchrotron radiation facilities.

In this project, we have probed the possibility to use the DuMond spectrometer for in-house EXAFS measurements, using the X-ray tube to produce the continuous energy $\mathrm{X}$-ray beam and the crystal of the spectrometer to monochromatize the bremsstrahlung transmitted through the absorber and to scan the Bragg angle across the absorption edge. The

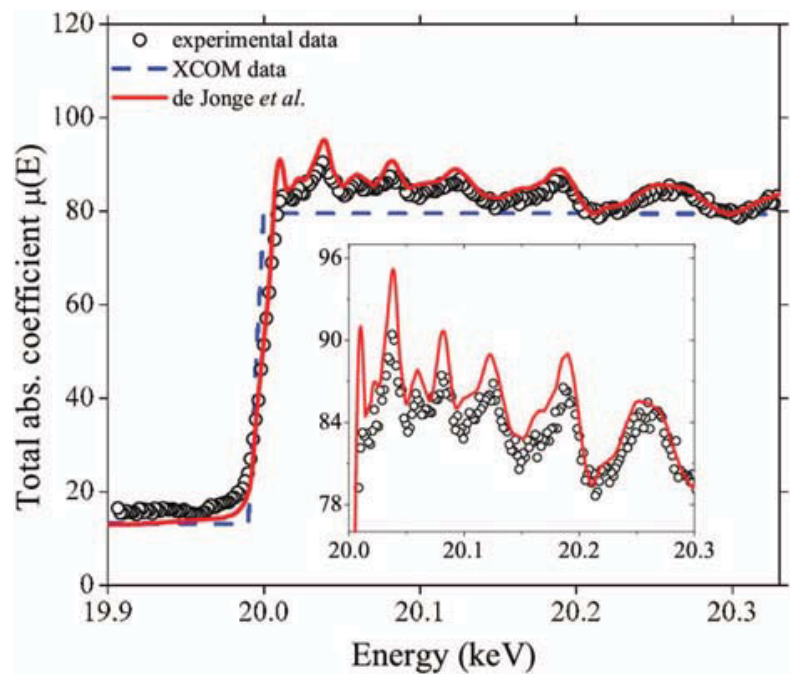

FIG. 11. Total mass attenuation coefficient of Mo around the $K$-edge in $\left(\mathrm{cm}^{2} / \mathrm{g}\right)$. The inset shows an enlarged view of the measured mass attenuation coefficients in the XANES and EXAFS regions. measurements were performed with an absorber consisting of a $\sim 20 \mu \mathrm{m}$ thick metallic foil of molybdenum positioned between the slit and the crystal. The absorption spectrum was observed around the $K$-edge which lies for Mo at 20000.5 $\pm 2.1 \mathrm{eV}^{39}$ In these measurements the Au anode X-ray tube was operated at $40 \mathrm{kV}$ and $50 \mathrm{~mA}$.

At first, the angular spectrum around the Bragg angle corresponding to the $K$ absorption edge of Mo was scanned without absorber. Then, the same measurement was repeated, but this time with the Mo foil fixed on the slit (width of $0.1 \mathrm{~mm}$ ). Both scans consisted of 250 points with acquisition times of $20 \mathrm{~s}$ per point in the first measurement and $200 \mathrm{~s}$ in the second one. The measurement with the absorber was performed on both sides of diffraction for the energy calibration of the spectrometer.

The X-ray mass attenuation coefficients $\mu(\mathrm{E})$ deduced from the intensities measured in the two scans are presented in Fig. 11 as a function of the photon energy. For comparison the values taken from the database $\mathrm{XCOM}^{45}$ and those obtained by de Jonge et al. ${ }^{46}$ in a synchrotron radiation-based experiment are also shown. The oscillations observed above the edge correspond to XANES and EXAFS structures. They are due to the scattering of the photoelectrons by neighboring atoms. The small dip observed below the edge, at about $19.96 \mathrm{keV}$, corresponds to the $\mathrm{K} \beta_{2} \mathrm{X}$-ray line of Mo. This fluorescence line is produced in the absorber by bremsstrahlung photons that have an energy higher than $20 \mathrm{keV}$.

In general our results for the absorption coefficient $\mu(\mathrm{E})$ are in good agreement with the semi-experimental XCOM data and especially with the SR data from Ref. 46. As the XCOM data correspond to so-called "atom-like" absorption coefficients (absorption coefficients for free and isolated atoms) they do not exhibit any XANES or EXAFS structure. In the case of the SR data the small differences in intensity with respect to our measurements can be explained by the natural oxidation of our sample and by inhomogeneities in the thickness of the employed absorber foil.

Absorption edge measurements provide the possibility to determine the binding energy of the atomic level corresponding to the measured edge. The binding energy of the Mo $1 \mathrm{~s}$ atomic level deduced from our measurements is 20000.18 $\pm 0.36 \mathrm{eV}$. This result is in good agreement with the value of $20000.351 \pm 0.020 \mathrm{eV}$ reported in Ref. 47 .

\section{SUMMARY}

In this paper the high-resolution curved crystal spectrometer of Fribourg based on the modified DuMond slit geometry was presented. The spectrometer was designed to measure photoinduced X-ray spectra of solid, liquid, and gaseous 
samples for photon energies above about $10 \mathrm{keV}$, i.e., for inhouse metrology measurements of $K$ and $L$ x-ray transitions of heavy elements. For a slit width of $0.1 \mathrm{~mm}$ the angular instrumental response of the instrument was found to be well reproduced by a Gaussian function with a standard deviation of $23 \mu \mathrm{rad}$. For the $3 \mathrm{rd}$ diffraction order of the Mo K $\alpha_{1} \mathrm{X}$-ray line $(\mathrm{E}=17.479 \mathrm{keV})$ this corresponds to a FWHM energy resolution of $2.2 \mathrm{eV}$, i.e., to a resolving power of about $10^{-4}$. Thanks to the determination of the Bragg angles by means of a Doppler shift based laser interferometer, it was shown that the energies of even weak X-ray transitions can be determined with an accuracy of about $10 \mathrm{ppm}$. The high-resolution, precision, sensitivity, and versatility of the instrument were discussed in detail and illustrated with typical examples of applications.

\section{ACKNOWLEDGMENTS}

The financial support of the Swiss National Science Foundation is acknowledged. The authors are also grateful to the mechanicians' and electronicians' staff of the Physics Department of the University of Fribourg for their technical assistance during the construction of the spectrometer.

${ }^{1}$ H. H. Johann, Z. Phys. 69, 185 (1931).

${ }^{2}$ T. Johansson, Z. Phys. 82, 507 (1933).

${ }^{3}$ L. von Hamos, Naturwissenschaften 20, 705 (1932).

${ }^{4}$ L. von Hamos, Ann. Phys. 409, 716 (1933).

${ }^{5}$ L. von Hamos, Ann. Phys. 411, 252 (1934).

${ }^{6}$ J. W. M. DuMond, Rev. Sci. Instrum. 18, 626 (1947).

${ }^{7}$ Y. Cauchois, J. Phys. Radium 3, 320 (1932).

${ }^{8}$ N. Ryde and B. Andersson, Proc. Phys. Soc. B 68, 1117 (1955).

${ }^{9}$ O. Beckman, P. Bergvall, and B. Axelsson, Ark. Fys. 14, 419 (1958).

${ }^{10}$ K. M. Crowe and R. E. Shafer, Rev. Sci. Instrum. 38, 1 (1967).

${ }^{11}$ O. Piller, W. Beer, and J. Kern, Nucl. Instrum. Methods 107, 61 (1973).

${ }^{12}$ G. L. Borchert, W. Scheck, and O. W. B. Schult, Nucl. Instrum. Methods 124, 107 (1975).

${ }^{13}$ H. R. Koch, H. G. Börner, J. A. Pinston, W. F. Davidson, J. Faudou, R. Roussille, and O. W. B. Schult, Nucl. Instrum. Methods 175, 401 (1980).

${ }^{14}$ G. L. Borchert, J. Bojowald, A. Ercan, H. Labus, T. Rose, and O. W. B. Schult, Nucl. Instrum. Methods Phys. Res. A 245, 393 (1986).

${ }^{15}$ B. Perny, J.-Cl. Dousse, M. Gasser, J. Kern, R. Lanners, C. Rhême, and W. Schwitz, Nucl. Instrum. Methods Phys. Res. A 267, 120 (1988).

${ }^{16}$ T. Ludziejewski, J. Hoszowska, P. Rymuza, Z. Sujkowski, D. Anagnostopoulos, G. L. Borchert, M. Carlen, J.-Cl. Dousse, C. Rhême, and A. Drentje, Nucl. Instrum. Methods Phys. Res. B 63, 494 (1992).

${ }^{17}$ J. Kern, A. Raemy, W. Beer, J.-Cl. Dousse, W. Schwitz, M. Balodis, P. Prokofjev, N. Kramer, L. Simonova, R. Hoff, D. Gardner, M. Gardner, R. Casten, R. Gill, R. Eder, T. von Egidy, E. Hagn, P. Hungerford, H. Scheerer, H. Schmidt, E. Zech, A. Chalupka, A. Murzin, V. Libman, I. Kononenko, C. Coceva, P. Giacobbe, I. Kondurov, Y. Loginov, P. Sushkov, S. Brant, and V. Paar, Nucl. Phys. A 534, 77 (1991).

${ }^{18}$ S. Mannanal, B. Boschung, M. Carlen, J.-Cl. Dousse, S. Drissi, P. Garrett, J. Kern, B. Perny, C. Rhême, J. Vorlet, C. Günthe, J. Manns, and U. Müller, Nucl. Phys. A 582, 141 (1995).

${ }^{19}$ B. Perny, J.-Cl. Dousse, M. Gasser, J. Kern, C. Rhême, P. Rymuza, Z. Sujkowski, and J. Vorlet, Phys. Rev. A 36, 2120 (1987).
${ }^{20}$ M. Carlen, M. Polasik, B. Boschung, J.-Cl. Dousse, M. Gasser, Z. Halabuka, J. Hoszowska, J. Kern, B. Perny, C. Rhême, P. Rymuza, and Z. Sujkowski, Phys. Rev. A 46, 3893 (1992).

${ }^{21}$ J. Rzadkiewicz, D. Chmielewska, Z. Sujkowski, J.-Cl. Dousse, D. Castella, D. Corminboeuf, J. Hoszowska, P.-A. Raboud, M. Polasik, K. Slabkowska, and M. Pajek, Phys. Rev. A 68, 032713 (2003).

${ }^{22}$ R. Eichler, B. Aas, W. Beer, I. Beltrami, P. Ebersold, T. von Ledebur, H. Leisi, W. Sapp, J.-Cl. Dousse, J. Kern, and W. Schwitz, Phys. Lett. B 76, 231 (1978).

${ }^{23}$ W. Beer, K. Bos, G. D. Chambrier, K. L. Giovanetti, P. F. A. Goudsmit, B. Grigoryev, B. Jeckelmann, L. Knecht, L. N. Kondurova, J. Langhans, H. J. Leisi, P. M. Levchenko, V. I. Marushenko, A. F. Mezentsev, H. Obermeier, A. A. Petrunin, U. Rohrer, A. Sergeev, S. G. Skornjakov, A. I. Smirnov, E. Steiner, G. Strassner, V. M. Suvorov, and A. Vacchi, Nucl. Instrum. Methods Phys. Res. A 238, 365 (1985).

${ }^{24}$ J. Hoszowska, J.-Cl. Dousse, J. Kern, and C. Rhême, Nucl. Instrum. Methods Phys. Res. A 376, 129 (1996).

${ }^{25}$ A. Hudson, W. Stolte, D. Lindle, and R. Guillemin, Rev. Sci. Instrum. 78, 053101 (2007).

${ }^{26}$ L. Journel, L. E. Khoury, T. Marin, R. Guillemin, S. Carniato, A. Avila, R. Delaunay, C. Haguue, and M. Simon, Rev. Sci. Instrum. 80, 093105 (2009).

${ }^{27}$ E. Kleymenov, J. A. van Bokhoven, C. David, P. Glatzel, M. Janousch, R. Alonso-Mori, M. Studer, M. Willimann, A. Bergamaschi, B. Henrich, and M. Nachtegaal, Rev. Sci. Instrum. 82, 065107 (2011).

${ }^{28}$ J. Szlachetko, M. Nachtegaal, E. de Boni, M. Willimann, O. Safonova, J. Sa, G. Smolentsev, M. Szlachetko, J. A. van Bokhoven, J.-Cl. Dousse, J. Hoszowska, Y. Kayser, P. Jagodzinski, A. Bergamaschi, B. Schmitt, C. David, and A. Lucke, Rev. Sci. Instrum. 83, 103105 (2012).

${ }^{29}$ I. Llorens, E. Lahera, W. Delnet, O. Proux, A. Braillard, J.-L. Hazemann, A. Prat, D. Testemale, Q. Dermigny, F. Gelebart, M. Morand, A. Shukla, N. Bardou, O. Ulrich, S. Arnaud, J.-F. Berar, N. Boudet, B. Caillot, P. Chaurand, J. Rose, E. Doelsch, P. Martin, and P. L. Solari, Rev. Sci. Instrum. 83, 063104 (2012).

${ }^{30}$ M. Kavčič, M. Budnar, A. Muhleisen, F. Gasser, M. Zitnik, K. Bučar, and R. Bohinc, Rev. Sci. Instrum. 83, 033113 (2012).

${ }^{31}$ D. Sokaras, D. Nordlund, T.-C. Weng, R. A. Mori, P. Velikov, D. Wenger, A. Garachtchenko, M. George, V. Borzenets, B. Johnson, Q. Qian, T. Rabedeau, and U. Bergmann, Rev. Sci. Instrum. 83, 043112 (2012).

${ }^{32}$ D. Sokaras, T.-C. Weng, D. Nordlund, R. Alonso-Mori, P. Velikov, D. Wenger, A. Garachtchenko, M. George, V. Borzenets, B. Johnson, T. Rabedeau, and U. Bergmann, Rev. Sci. Instrum. 84, 053102 (2013).

${ }^{33}$ N. G. Kujala, C. Karanfil, and R. A. Barrea, Rev. Sci. Instrum. 82, 063106 (2011).

${ }^{34}$ N. Hiraoka, H. Fukui, H. Tanida, H. Toyokawa, Y. Q. Cai, and K. D. Tsuei, J. Synchrotron Radiat. 20, 266 (2013).

${ }^{35}$ Y.-P. Maillard, J.-Cl. Dousse, and J. Hoszowska, Eur. Phys. J. D 57, 155 (2010).

${ }^{36}$ W. Schwitz, Nucl. Instrum. Methods 154, 95 (1978).

${ }^{37}$ Moore Rotary Table, Type 7046, Moore special tool Co., Inc. Bridgeport, Connecticut, USA.

${ }^{38}$ Technical specification, PCI-6040 E series Multifunctional I/O card, National Instruments, Austin, USA.

${ }^{39}$ R. D. Deslattes, E. G. Kessler, J. P. Indelicato, L. de Billy, E. Lindroth, and J. Anton, Rev. Mod. Phys. 75, 35 (2003).

${ }^{40}$ PeakFit $^{\mathrm{TM}}$, Peak Separation and Analysis Software, User's manual, SYSTAT Software Inc., Richmond, CA 94904-2028, USA.

${ }^{41}$ J. L. Campbell and T. Papp, At. Data Nucl. Data Tables 77, 1 (2001).

${ }^{42}$ B. Galley and J.-Cl. Dousse, Phys. Rev. A 50, 3058 (1994).

${ }^{43}$ J. H. Scofield, At. Data Nucl. Data Tables 14, 121 (1974).

${ }^{44}$ E. G. Kessler, R. D. Deslattes, D. Girard, W. Schwitz, L. Jacobs, and O. Renner, Phys. Rev. A 26, 2696 (1982).

${ }^{45}$ See http://physics.nist.gov/PhysRefData/Xcom/Text/XCOM.html for Photon Cross Sections Database.

${ }^{46}$ M. D. de Jonge, C. Q. Tran, C. T. Chantler, Z. Barnea, B. B. Dhal, D. J. Cookson, W.-K. Lee, and A. Mashayekhi, Phys. Rev. A 71, 032702 (2005).

${ }^{47}$ S. Kraft, J. Stumpel, P. Becker, and U. Kuetgens, Rev. Sci. Instrum. 67, 681 (1996). 